\title{
Arte Marcial Chinesa: Cultura e Movimento
}

\author{
Walter Roberto Correia
}

Escola de Educação Física e Esporte da Universidade de São Paulo

* Autor para correspondência:

\section{RESUMO}

A Universidade de São Paulo oferece uma disciplina de graduação denominada Arte Marcial Chinesa: Cultura e Movimento, que visa a introduzir e a promover o sistema Ving Tsun. O objetivo do presente estudo foi verificar o efeito de um programa Ving Tsun/Kung Fu sobre o conhecimento declarativo de estudantes de graduação da Escola de Educação Física e Esporte da Universidade de São Paulo (EEFEUSP). Quarenta estudantes de graduação participaram de um programa Ving Tsun/Kung Fu de dezesseis semanas realizado na EEFEUSP. $\mathrm{O}$ conhecimento declarativo dos alunos foi analisado por meio de registros orais, dissertações e entrevistas. A análise de conteúdo foi baseada no método de Bardin. Os resultados presentes sugerem que um programa Ving Tsun/Kung Fu pode fornecer mudanças nos conceitos prévios de estudantes de graduação em relação à sua visão sobre o uso de habilidades técnicas de Ving Tsun/Kung Fu, que se deslocam da percepção de usá-las apenas com fins de combate para o uso como ferramenta de desenvolvimento humano.

Palavras-Chave: Arte Marcial Chinesa; Cultura; Movimento.

\begin{abstract}
The University of São Paulo offers a undergraduate subject named as Chinese Martial Art: culture and movement, which aims to introduce and promote the Ving Tsun system. The aim of the present study was to verify the effect of a Ving Tsun/Kung Fu program on the declarative knowledge of undergraduate students from the School of Physical Education and Sport, University of São Paulo. Forty undergraduate students participated in a 16-week Ving Tsun/Kung Fu program conducted at EEFEUSP. The declarative knowledge of the students was analysed by oral records dissertations interviews. The content analysis was based on Bardin's method. The present results suggest that Ving Tsun/Kung Fu program may provide changes in previous concepts of undergraduate students regard their view about the use of Ving Tsun/Kung Fu technical skills shifting from the perception of using them just for fighting purposes to their use as a tool for the human development.
\end{abstract}

Keywords: Chinese Martial Art; Culture Movement.

As artes marciais representam um conjunto de manifestações culturais marcadas por práticas e sistemas de conhecimento muito diversos, perpassando os diferentes momentos históricos do processo civilizatório. A força desse fenômeno pode ser percebida nas mais diferentes instâncias da vida social e cotidiana. No Brasil, as lutas, artes marciais e modalidades esportivas de combate são reconhecidas pela sua pluralidade, dinamismo e pela presença e atuação arrojada de interlocutores de reconhecimento internacional. Dessa forma, as artes marciais compreendidas como um fenômeno antropológico dotado de relevância e especificidade estão cada vez mais sendo assimiladas como objetos de intervenção acadêmica e profissional da Educação Física e do Esporte (CORREIA \& FRANCHINI, 2010). Desde o ano de 2011, a Escola de Educação Física e Esporte da USP oferece uma disciplina curricular denominada Arte Marcial Chinesa: Cultura e Movimento, cujo propósito é o de promover uma introdução da arte marcial "Ving Tsun" (Kung Fu) aos estudantes universitários.

Esclarecendo melhor os termos provenientes da cultura chinesa, Kung Fu é o termo mais popularizado que faz referência às lutas e artes marciais chinesas. Essa ideia foi amplamente disseminada por meio do cinema internacional e chegou ao Brasil 
por intermédio dos imigrantes chineses na década de 50 do século passado. O Kung Fu é toda manifestação humana, cuja habilidade, maestria ou excelência foi forjada a partir do esforço pessoal. Assim sendo, podemos admitir e testemunhar que algum cidadão ou cidadã tem Kung Fu nas diversas atividades humanas do cotidiano e, por sua vez, não se circunscreve exclusivamente nas técnicas e artes de combate (CHENG, 2008). Se uma habilidade proeminente for expressão de algo nato, ou seja, um dom, não se trata de considerá-la como Kung $\mathrm{Fu}$, uma vez que o emprego do esforço pessoal é o critério distintivo do conceito associado à atitude de "cultivo" e "elaboração" das energias psíquicas e somáticas integradas.

O Ving Tsun é uma forma tradicional de Kung Fu oriunda do sul da China e pode ser considerada como um sistema chinês de inteligência estratégica, cuja fundação é atribuída à Yim Ving Tsun (IMAMURA, 1994). O Ving Tsun é uma das raras artes marciais cujo protagonismo de origem foi atribuído a uma mulher. Ving significa em cantonês chamar, anunciar e cantar; Tsun significa primavera, renovação e mudança. O símbolo da arte remete-se à imagem da flor de ameixeira. Essa flor está investida de alguns simbolismos, uma vez que representa o feminino, a China e seus principais grupos étnicos e a própria arte marcial. O Ving Tsun é constituído por seis domínios de estudos e experiências, subsidiado por listagens ou sequências de movimentos predefinidos para cada nível, de forma a fornecer os conceitos e as habilidades necessários para o desenvolvimento humano na arte marcial.

A linguagem de base que sustenta o processo de apropriação e expressão da arte é a linguagem corporal, mais especificamente a linguagem de combate corporal simbólico. Simbólico, uma vez que a experiência marcial de combate e a arte de lutar precisam ser mediadas por limites éticos e estratégicos, para que se possam favorecer o desenvolvimento e a integridade de seus praticantes e estudiosos. Se tudo valesse na experiência marcial, limitaríamos consideravelmente o estudo, a apreciação, a análise, a compreensão, a inserção e, principalmente, a aprendizagem de muitos praticantes.
O Ving Tsun é considerado um sistema, pois seus princípios e conteúdos devem manter relações recíprocas entre si em termos de potencial de significação. As experiências de combate simbólico devem ensejar o cultivo da inteligência estratégica, ou, dito de outro modo, contribuir para o desenvolvimento da capacidade de antecipação de um benefício em potencial em diversas situações da existência, seja no combate corporal, seja na vida cotidiana (FAYARD, 2006; JULLIEN, 1998, 2000, 2017). Nesse caso, é fundamental que a resultante experiencial seja estendida para o contexto de vida dos praticantes ou estudiosos. Tudo isso deve ser permeado pela relação tutorial estabelecida entre um mestre e um discípulo, uma vez que o propósito não é ensinar técnicas de Kung Fu e, sim, Kung Fu. Para que isso ocorra, temos o que denominamos de "Vida Kung Fu".

Vida Kung Fu é a relação vivida entre mestre e discípulo para além dos limites do recinto de treino ou escola de arte marcial, tomando vários momentos e contextos em potencial para a extrapolação da inteligência estratégica e do próprio Kung Fu, ou seja, experimentar o Kung Fu no fluxo da vida em diferentes locais e tempos do dia a dia. É importante esclarecer que esse entendimento se inscreve numa formação tradicional da arte e, dessa maneira, não é o nosso propósito viabilizar essa forma de estudo e conduta na Universidade de São Paulo. No entanto, faz parte da proposta da disciplina em questão (Arte Marcial Chinesa, Cultura e Movimento) apresentar a ideia aos interessados, sobretudo pela força simbólica desses expedientes e conteúdos.

Entre as clássicas funções da universidade, temos o entendimento de que pertencem aos domínios acadêmicos o investimento na preservação do patrimônio cultural e universal da humanidade nas suas múltiplas formas de expressão e, especialmente, o estímulo aos intercâmbios dos bens culturais entre os povos. Assim sendo, a partir do oferecimento da referida disciplina, foi possível analisar os significados apresentados pelos estudantes universitários após se submeterem a um programa introdutório do Ving Tsun Kung Fu no que diz 
respeito às suas qualidades distintivas. Para tanto, partindo de uma abordagem de natureza qualitativa (CHIZZOTTI, 1991), foi viabilizada uma análise de conteúdo (BARDIN, 1979) de forma a propiciar uma interpretação hermenêutica dos sentidos e significados apreendidos pelos estudantes sem o estabelecimento de categorias a priori. Os dados foram obtidos das expressões orais registradas em diário no início e ao final de cada unidade de aula, além das dissertações desenvolvidas pelos discentes e colaboradores ao final da disciplina como trabalho de conclusão.

Como resultados, foi possível identificarmos elementos simbólicos na expressão dos sujeitos da pesquisa no que diz respeito à: 1) Reelaboração do conceito da prática de luta para estudo de combate simbólico; 2) Indicação de mudança na noção do movimento corporal como técnica para dispositivo corporal; 3) Ampliação da concepção de instrutor para tutor de aprendizagens significativas; 4) Incorporação do conceito de desenvolvimento da inteligência estratégica por meio da experiência marcial, extensivo para o desenvolvimento humano em geral. Esses resultados foram apresentados em julho de 2014 (Holanda/Amsterdam) no European College of Sport Science, sob o título "Chinese Martial Art's Program at the Brazilian University".

\section{Referências Bibliográficas}

BARDIN, L. Análise de Conteúdo. Lisboa: Ed. 70, 1979.

CHENG, A. História do Pensamento Chinês. Petrópolis: Vozes, 2008.

CHIZZOTTI, A. Pesquisa em Ciências Humanas e Sociais. São Paulo: Cortez, 1991.

CORREIA, W. R. \& FRANCHINI, E. "Produção Acadêmica em Lutas, Artes Marciais e Modalidades Esportivas de Combate". Revista Motriz, Rio Claro/SP, vol. 16, n. 1, 2010.

FAYARD, P. Compreender e Aplicar Sun Tzu; o Pensamento Estratégico Chinês: uma Sabedoria em Ação. Porto Alegre: Bookman, 2006.

IMAMURA, L. Ving Tsun Biu fe. São Paulo: Biopress Editora, 1994.

JULLIEN, F. Tratado da Efccácia. São Paulo: Editora 34, 1998. Fontes, 2000.

Um Sábio não Tem Ideias. São Paulo: Martins . A Propensão das Coisas: por uma Historia da Eficácia da China. São Paulo: Editora Unesp, 2017.

Publicado em 22/12/2017. 